\title{
FINITE ELEMENT APPROXIMATION OF THE PARABOLIC $p$-LAPLACIAN*
}

\author{
JOHN W. BARRETT ${ }^{\dagger}$ AND W. B. LIU ${ }^{\dagger}$
}

\begin{abstract}
In this paper the authors consider the continuous piecewise linear finite element approximation in space of the following problem:

Given $p \in(1, \infty), f$ and $u_{0}$; find $u$ such that

$$
\begin{aligned}
u_{t} & =\nabla \cdot\left(|\nabla u|^{p-2} \nabla u\right)+f \text { in } \Omega \times(0, T], \\
u & =0 \text { on } \partial \Omega \times(0, T], \\
u(x, 0) & =u_{0}(x) \quad \forall x \in \Omega,
\end{aligned}
$$

where $\Omega \subset \mathbf{R}^{d}, d=1$ or 2 .

The authors analyse the semidiscrete approximation and a fully discrete approximation using the backward Euler time discretisation, obtaining error bounds which improve on those in the literature.
\end{abstract}

Key words. finite elements, error analysis, parabolic $p$-Laplacian, degenerate equations

AMS subject classifications. 65M15, 65M60, 35K65

1. Introduction. Let $\Omega$ be a bounded domain in $\mathbf{R}^{d}, d=1$ or 2 with a Lipschitz boundary in the case $d=2$. Given $p \in(1, \infty), f$, and $u_{0}$, we consider the following problem-the parabolic $p$-Laplacian:

Find $u$ such that

$$
\begin{aligned}
u_{t} & =\nabla \cdot\left(|\nabla u|^{p-2} \nabla u\right)+f \text { in } \Omega \times(0, T] \\
u & =0 \text { on } \partial \Omega \times(0, T],
\end{aligned}
$$

and

$$
u(x, 0)=u_{0}(x) \quad \forall x \in \Omega
$$

This problem occurs in many mathematical models of physical processes: for example, nonlinear diffusion and filtration, see [13], and non-Newtonian flows, see [1]. It is the purpose of this paper to analyse the continuous piecewise linear finite element approximation of the above problem. Of course, one can study more general boundary conditions, the presence of lower-order terms in the differential operator as well as higher space dimensions. However, for ease of exposition we consider just the problem outlined above, even though most of our results can be adapted to more general problems. We note that due to the degenerate nature of the elliptic operator if $p \neq 2$, which leads to limited regularity of the solution $u$, see $\S 6$, there is little point in using higher-order elements. Glowinski and Marrocco [6] analysed the steady state case. With $u^{h}$ denoting the finite element approximation and assuming $u \in W^{2, p}(\Omega)$, they proved that $\left\|u-u^{h}\right\|_{W^{1, p}(\Omega)}$ was bounded above by $C h^{\alpha}$, where $\alpha=1 /(3-p)$ if $p \in(1,2]$ and $\alpha=1 /(p-1)$ if $p \in[2, \infty)$. Under the same assumptions, these error bounds were improved to $\alpha=p / 2$ if $p \in(1,2]$ and $\alpha=2 / p$ if $p \in[2, \infty)$, by exploiting the asssociated minimisation properties for $u$ and $u^{h}$ by Tyukhtin [15]and Chow [5]. Recently in [2] we proved an optimal, $C h$, error bound for $\left\|u-u^{h}\right\|_{W^{1, p}(\Omega)}$ in the case

* Received by the editors August 19, 1992; accepted for publication (in revised form) April 12 1993.

$\dagger$ Department of Mathematics, Imperial College, London, United Kingdom, SW7 2BZ (jbarrett@ic.ac.uk; w.liu@ee.surrey.ac.uk).

$\ddagger$ The research of this author was supported by Science and Engineering Research Council of U.K. grant GR/F81255. 
$p \in(1,2]$ under the stronger regularity requirement $u \in W^{3,1}(\Omega) \cap C^{2,(2-p) / p}(\bar{\Omega})$. For the case $p \in(2, \infty)$ we proved the Tyukhtin [15] and Chow [5] result, under the relaxed regularity requirement $u \in H^{2}(\Omega) \cap W^{1, \infty}(\Omega)$. If in addition $f \in L^{\infty}(\Omega)$ and $|f| \geq \beta$ for some positive constant $\beta$, we proved in [2] an optimal, $C h$, bound for $\left\|u-u^{h}\right\|_{W^{1,1}(\Omega)}$. See $\S 6$ for conditions on the data to ensure that the above regularity requirements on $u$ are achieved.

The only paper, to our knowledge, that analyses the discretisation error for the parabolic problem is Wei [16]. In this paper it is shown that the backward Euler discretisation in time-continuous piecewise linear finite elements in space approximation (see $\left(P^{h, \Delta t}\right)$ in $\left.\S 4\right)$ is such that for $n=1 \rightarrow N$ with $N \Delta t=T$

$$
\left|u(\cdot, n \Delta t)-U^{n}(\cdot)\right|_{L^{2}(\Omega)} \leq C\left[h^{1 / 2(p-1)}+(\Delta t)^{1 / 2}\right],
$$

under the assumptions $p \in(2, \infty), u \in C\left([0, T] ; W^{2, p}(\Omega)\right)$ and $f:[0, T] \rightarrow L^{2}(\Omega)$ is Lipschitz continuous.

It is the purpose of this paper to improve on this error bound. For example, under weaker regularity assumptions on $u$ we can replace $h^{1 / 2(p-1)}$ on the right-hand side of $(1)$ by $h$. In addition, we extend this result to the case $p \in(1,2)$ and prove error bounds for the gradient of $u$.

Throughout this paper we adopt the standard notation $W^{m, q}(D)$ for Sobolev spaces on a domain $D$ with norm $\|\cdot\|_{W^{m, q}(D)}$ and seminorm $|\cdot|_{W^{m, q}(D)}$. We note that $|\cdot|_{W^{1, q}(D)}$ and $\|\cdot\|_{W^{1, q}(D)}$ are equivalent on $W_{0}^{1, q}(D) \equiv\left\{v \in W^{1, q}(D): v=0\right.$ on $\left.\partial D\right\}$. When $q=2$ we write $W^{m, 2}(D) \equiv H^{m}(D)$. We denote by $L^{s}\left(0, T ; W^{m, q}(D)\right)$ the Banach space of $L^{s}$ functions from $(0, T)$ into $W^{m, q}(D)$ with norm $\|v\|_{L^{s}\left(0, T ; W^{m, q}(D)\right)} \equiv$ $\left(\int_{0}^{T}\|v(t)\|_{W^{m, q}(D)}^{s} d t\right)^{1 / s}$ for $s \in[1, \infty)$ with the standard modification for $s=\infty$. We will often write $|v|_{L^{s}\left(0, T ; W^{m, q}(D)\right)}$ to denote $\left(\int_{0}^{T}|v(t)|_{W^{m, q}(D)}^{s} d t\right)^{1 / s}$. Similarly, one can define $H^{1}\left(0, T ; W^{m, q}(D)\right)$ and $C^{k}\left([0, T] ; W^{m, q}(D)\right)$ (see, for example, [10]). Throughout, $C$ denotes a generic positive constant independent of the mesh parameter $h$. In some cases the parameters on which $C$ depends may be displayed as arguments.

The layout of this paper is as follows: In the next section we study the weak formulation of the parabolic $p$-Laplacian and gather together some key results for later use. In $\S 3$ we prove an abstract error bound for the continuous-in-time finite element approximation (semidiscrete). In $\S 4$ we prove an abstract error bound for the fully discrete approximation using the backward Euler discretisation in time. In $\S 5$ we deduce explicit error bounds for these approximations under certain regularity requirements on $u$. Finally, in $\S 6$ we discuss these regularity requirements.

2. Preliminaries. We make the following assumptions on the data:

$$
f \in C\left([0, T] ; L^{2}(\Omega)\right) \text { and } u_{0} \in W_{0}^{1, p}(\Omega) .
$$

Setting $V \equiv W_{0}^{1, p}(\Omega)$, the weak formulation of the parabolic $p$-Laplacian problem is then as follows:

$(P)$ Find $u \in L^{\infty}(0, T ; V) \cap H^{1}\left(0, T ; L^{2}(\Omega)\right)$ such that for almost every $t \in(0, T]$

$$
\begin{aligned}
\int_{\Omega} u_{t} v+\int_{\Omega}|\nabla u|^{p-2} \nabla u . \nabla v & =\int_{\Omega} f v \quad \forall v \in V, \\
u(0) & =u_{0} .
\end{aligned}
$$

It is a simple matter to show that under assumptions $(A)$ there exists a unique solution to $(P)$ and from imbedding $u \in C\left([0, T] ; L^{2}(\Omega)\right)$. For example, one can 
adapt the argument in [16], where it is shown that if, in addition to $(A) f$ is Lipschitz continuous in time and $\nabla \cdot\left(\left|\nabla u_{0}\right|^{p-2} \nabla u_{0}\right) \in L^{2}(\Omega)$, then there exists a unique solution to $(P)$ for $p \in(2, \infty), u \in C([0, T] ; V)$, and $u_{t} \in C\left([0, T] ; L^{2}(\Omega)\right)$. We have the following stability result.

LEMMA 2.1. Under assumptions $(A)$ the unique solution $u$ of $(P)$ is such that

$$
\begin{aligned}
& \left|u_{t}\right|_{L^{2}\left(0, T ; L^{2}(\Omega)\right)}^{2}+|u|_{L^{\infty}\left(0, T ; W^{1, p}(\Omega)\right)}^{p} \\
& \quad \leq C(p)\left[|f|_{L^{2}\left(0, T ; L^{2}(\Omega)\right)}^{2}+\left|u_{0}\right|_{W^{1, p}(\Omega)}^{p}\right] .
\end{aligned}
$$

Proof. The result (2) follows immediately by choosing $v \equiv u_{t}$ in $(P)$, in a distributional sense, integrating in time over $(0, s), s \in(0, T]$; and noting that

$$
\frac{d}{d t}\left[\int_{\Omega}|\nabla u|^{p}\right] \equiv p \int_{\Omega}|\nabla u|^{p-2} \nabla u . \nabla u_{t} .
$$

We now state two lemmas, both of which are the key to our improved error bounds. The first one is proved in [2]. However, we give a proof for completeness.

Lemma 2.2. For all $p \in(1, \infty)$ and $\delta \geq 0$ there exist positive constants $C_{1}(p, d)$ and $C_{2}(p, d)$ such that for all $\xi, \eta \in \mathbf{R}^{d}, d \geq 1$,

$$
\left.|| \xi\right|^{p-2} \xi-|\eta|^{p-2} \eta\left|\leq C_{1}\right| \xi-\left.\eta\right|^{1-\delta}(|\xi|+|\eta|)^{p-2+\delta}
$$

and

$$
\left(|\xi|^{p-2} \xi-|\eta|^{p-2} \eta\right) \cdot(\xi-\eta) \geq C_{2}|\xi-\eta|^{2+\delta}(|\xi|+|\eta|)^{p-2-\delta}
$$

Proof. We first prove (3) with $\delta=0$. For any $\xi, \eta \in \mathbf{R}^{d}$ let

$$
F(\xi, \eta) \equiv \frac{\left.|| \xi\right|^{p-2} \xi-|\eta|^{p-2} \eta \mid}{|\xi-\eta|(|\xi|+|\eta|)^{p-2}}
$$

We wish to prove that $F$ is bounded. For any $\epsilon>0 F$ is continuous on $D_{\epsilon} \equiv\{(\xi, \eta) \epsilon$ $\mathbf{R}^{d} \times \mathbf{R}^{d}:|\xi-\eta| \geq \epsilon$ and $\left.|\xi|+|\eta| \leq \epsilon^{-1}\right\}$. Furthermore, with $A$ a $d \times d$ orthogonal matrix it follows that for all $(\xi, \eta) \in \mathbf{R}^{d} \times \mathbf{R}^{d}$

$$
F(\xi, \eta)=F(\eta, \xi), \quad F(A \xi, A \eta)=F(\xi, \eta) \quad \text { and } \quad F(0, \eta) \leq C .
$$

Therefore, without loss of generality we can suppose that $\xi, \eta \neq 0$. Then $F$ can be rewritten as

$$
F(\xi, \eta) \equiv \frac{\left|\frac{\xi}{|\xi|}-\frac{\eta}{|\eta|} \frac{|\eta|^{p-2}}{|\xi|^{p-2}}\right|}{\left|\frac{\xi}{|\xi|}-\frac{\eta}{|\xi|}\right|\left(1+\frac{|\eta|}{|\xi|}\right)^{p-2}}
$$

We can further assume from (5) that $\xi /|\xi|=e_{1} \equiv(1,0, \ldots, 0)^{T}$ and $|\eta| /|\xi| \leq 1$. It follows that $F(\xi, \eta)$ will be bounded if $\eta /|\xi|$ does not tend to $e_{1}$. It remains to show that $\lim \sup _{\eta /|\xi| \rightarrow e_{1}} F(\xi, \eta)<\infty$.

If $1-\epsilon \leq|\eta| /|\xi| \leq 1$ for some $\epsilon \in(0,1)$, then there exists a constant $C$ such that $|\xi||\eta| \leq(|\xi|+|\eta|)^{2} \leq C|\xi||\eta|$. Then it follows, since $|\xi||\eta|-\xi . \eta \leq|\xi-\eta|^{2}$, that

$$
\begin{aligned}
\left.|| \xi\right|^{p-2} \xi-\left.|\eta|^{p-2} \eta\right|^{2} & =\left(|\xi|^{p-1}-|\eta|^{p-1}\right)^{2}+2|\xi|^{p-2}|\eta|^{p-2}(|\xi||\eta|-\xi . \eta) \\
& \leq C|\xi-\eta|^{2}(|\xi|+|\eta|)^{2(p-2)}
\end{aligned}
$$


Consequently, one has that (3) holds for $\delta=0$. On the other hand for all $\xi, \eta \in \mathbf{R}^{d}$ and $\delta \geq 0$ we have that

$$
|\xi-\eta|(|\xi|+|\eta|)^{p-2} \leq|\xi-\eta|^{1-\delta}(|\xi|+|\eta|)^{p-2+\delta}
$$

that is, (3) holds for all $\delta \geq 0$.

Similarly, (4) holds for $\delta=0$, since there exists a constant $C$ such that

$$
\begin{aligned}
\left(|\xi|^{p-2}\right. & \left.\xi-|\eta|^{p-2} \eta\right) \cdot(\xi-\eta) \\
= & \left(|\xi|^{p-1}-|\eta|^{p-1}\right)(|\xi|-|\eta|)+2\left(|\xi|^{p-2}+|\eta|^{p-2}\right)(|\xi||\eta|-\xi . \eta) \\
& \geq C\left[(|\xi|-|\eta|)^{2}+2(|\xi||\eta|-\xi . \eta)\right]\left(|\xi|^{p-2}+|\eta|\right)^{p-2} \\
= & C|\xi-\eta|^{2}(|\xi|+|\eta|)^{p-2} .
\end{aligned}
$$

In addition we have for all $\xi, \eta \in \mathbf{R}^{d}$ and $\delta \geq 0$ that

$$
|\xi-\eta|^{2}(|\xi|+|\eta|)^{p-2} \geq|\xi-\eta|^{2+\delta}(|\xi|+|\eta|)^{p-2-\delta}
$$

and hence (4) holds for all $\delta \geq 0$.

Remark 2.1. The inequality (3) was proved in [6] for $p \in(1,2]$ with $\delta=2-p$ and for $p \in[2, \infty)$ with $\delta=0$; similarly, (4) for $p \in(1,2]$ with $\delta=0$, and for $p \in[2, \infty)$ with $\delta=p-2$. This version of the above lemma was used by Wei [16] to produce the error bound (1). Our generalisation plays a key role in obtaining our improved error bounds.

Lemma 2.3. For all $p \in(1, \infty)$ there exists an $\epsilon_{0}$ such that for all $a, \sigma_{1}, \sigma_{2} \geq 0$ and for all $\epsilon \in\left(0, \epsilon_{0}\right)$

$$
\left(a+\sigma_{1}\right)^{p-2} \sigma_{1} \sigma_{2} \leq \epsilon\left(a+\sigma_{1}\right)^{p-2} \sigma_{1}^{2}+C\left(\epsilon^{-1}\right)\left(a+\sigma_{2}\right)^{p-2} \sigma_{2}^{2} .
$$

Proof. Consider first the case $p \in(1,2]$. Then we have that

$$
\left(a+\sigma_{1}\right)^{p-2} \sigma_{1} \sigma_{2} \leq\left(a+\sigma_{2}\right)^{p-2} \sigma_{2}^{2} \quad \text { for } \quad 0 \leq \sigma_{1} \leq \sigma_{2},
$$

since $(a+\sigma)^{p-2} \sigma$ is monotonically increasing for $\sigma \geq 0$. On the other hand,

$$
\begin{aligned}
\left(a+\sigma_{1}\right)^{p-2} \sigma_{1} \sigma_{2} & \leq\left(a+\sigma_{1}\right)^{(p-2) / 2} \sigma_{1}\left(a+\sigma_{2}\right)^{(p-2) / 2} \sigma_{2} \\
& \leq\left[\epsilon\left(a+\sigma_{1}\right)^{p-2} \sigma_{1}^{2}+\epsilon^{-1}\left(a+\sigma_{2}\right)^{p-2} \sigma_{2}^{2}\right] / 2 \\
& \text { for } 0 \leq \sigma_{2} \leq \sigma_{1} \text { and } \forall \epsilon>0 .
\end{aligned}
$$

Hence the desired result (6) holds for $p \in(1,2]$.

We now consider the case $p>2$. Clearly, (7) still holds. On the other hand, we have that for sufficiently small $\gamma(\epsilon, p)$

$$
\begin{aligned}
\left(a+\sigma_{1}\right)^{p-2} \sigma_{1} \sigma_{2} & \leq 2^{p-2}\left(\gamma^{1 /(p-1)} \sigma_{1}\right)^{p-1}\left(\gamma^{-1} \sigma_{2}\right) \\
& \leq 2^{p-2}\left[(p-1)\left(\gamma^{1 /(p-1)} \sigma_{1}\right)^{p}+\left(\gamma^{-1} \sigma_{2}\right)^{p}\right] / p \\
& \leq \epsilon\left(a+\sigma_{1}\right)^{p-2} \sigma_{1}^{2}+C\left(\epsilon^{-1}\right)\left(a+\sigma_{2}\right)^{p-2} \sigma_{2}^{2} \\
& \quad \text { for } 0 \leq a \leq \sigma_{1},
\end{aligned}
$$

and

$$
\begin{aligned}
\left(a+\sigma_{1}\right)^{p-2} \sigma_{1} \sigma_{2} & \leq 2^{p-2}\left(\gamma a^{(p-2) / 2} \sigma_{1}\right)\left(\gamma^{-1} a^{(p-2) / 2} \sigma_{2}\right) \\
& \leq 2^{p-3}\left(\gamma a^{(p-2) / 2} \sigma_{1}\right)^{2}+\left(\gamma^{-1} a^{(p-2) / 2} \sigma_{2}\right)^{2} \\
& \leq \epsilon\left(a+\sigma_{1}\right)^{p-2} \sigma_{1}^{2}+C\left(\epsilon^{-1}\right)\left(a+\sigma_{2}\right)^{p-2} \sigma_{2}^{2} \\
& \quad \text { for } 0 \leq \sigma_{1} \leq a .
\end{aligned}
$$

Hence the desired result $(6)$ holds for $p \in(2, \infty)$. 
3. Semidiscrete approximation. Let $V^{h}$ be a finite dimensional subspace of $V$. Then the corresponding semidiscrete approximation to $(P)$ is:

$\left(P^{h}\right)$ Find $u^{h} \in H^{1}\left(0, T ; V^{h}\right)$ such that for almost every $t \in(0, T]$

$$
\begin{aligned}
\int_{\Omega} u_{t}^{h} v^{h}+\int_{\Omega}\left|\nabla u^{h}\right|^{p-2} \nabla u^{h} \cdot \nabla v^{h} & =\int_{\Omega} f v^{h} \quad \forall v^{h} \in V^{h}, \\
u^{h}(0) & =u_{0}^{h},
\end{aligned}
$$

where $u_{0}^{h} \in V^{h}$ is an approximation to $u_{0}$.

It is easy to establish the existence of a unique solution to $\left(P^{h}\right)$ and that $u^{h} \in$ $C^{1}\left([0, T] ; V^{h}\right)$ under the assumptions $(A)$ by adapting the argument for $(P)$, i.e., the argument in [16]; and we have also the following analogue of Lemma2.1.

LEMMA 3.1. Under assumptions $(A)$ the unique solution $u^{h}$ of $\left(P^{h}\right)$ is such that

$$
\begin{aligned}
& \left|u_{t}^{h}\right|_{L^{2}\left(0, T ; L^{2}(\Omega)\right)}^{2}+\left|u^{h}\right|_{L^{\infty}\left(0, T ; W^{1, p}(\Omega)\right)}^{p} \\
& \quad \leq C(p)\left[|f|_{L^{2}\left(0, T ; L^{2}(\Omega)\right)}^{2}+\left|u_{0}^{h}\right|_{W^{1, p}(\Omega)}^{p}\right] .
\end{aligned}
$$

THEOREM 3.2. Under assumptions $(A)$ the unique solutions $u$ and $u^{h}$ of $(P)$ and $\left(P^{h}\right)$, respectively, are such that for any $v^{h} \in L^{q}\left(0, T ; V^{h}\right), q=\max (p, 2)$,

$$
\begin{aligned}
& \left|u-u^{h}\right|_{C\left([0, T] ; L^{2}(\Omega)\right)}^{2}+\int_{0}^{T} \int_{\Omega}\left[|\nabla u|+\left|\nabla\left(u-u^{h}\right)\right|\right]^{p-2}\left|\nabla\left(u-u^{h}\right)\right|^{2} \\
& \leq C \int_{0}^{T} \int_{\Omega}\left[|\nabla u|+\left|\nabla\left(u-v^{h}\right)\right|\right]^{p-2}\left|\nabla\left(u-v^{h}\right)\right|^{2}+C\left|u_{0}-u_{0}^{h}\right|_{L^{2}(\Omega)}^{2} \\
& \quad+C\left(\left|u_{0}^{h}\right|_{W^{1, p}(\Omega)}\right)\left|u-v^{h}\right|_{L^{2}\left(0, T ; L^{2}(\Omega)\right)} .
\end{aligned}
$$

Proof. Setting $e \equiv u-u^{h}$ we have from $(P)$ and $\left(P^{h}\right)$ that for any $v^{h} \in$ $L^{2}\left(0, T ; V^{h}\right)$ and for any $s \in(0, T]$

$$
\begin{aligned}
\frac{1}{2}|e(s)|_{L^{2}(\Omega)}^{2} & +\int_{0}^{s} \int_{\Omega}\left(|\nabla u|^{p-2} \nabla u-\left|\nabla u^{h}\right|^{p-2} \nabla u^{h}\right) \cdot \nabla e \\
\equiv & \int_{0}^{s} \int_{\Omega}\left(|\nabla u|^{p-2} \nabla u-\left|\nabla u^{h}\right|^{p-2} \nabla u^{h}\right) \cdot \nabla\left(u-v^{h}\right) \\
& +\int_{0}^{s} \int_{\Omega} e_{t}\left(u-v^{h}\right)+\frac{1}{2}\left|u_{0}-u_{0}^{h}\right|_{L^{2}(\Omega)}^{2} .
\end{aligned}
$$

Noting that for all $\xi, \eta \in \mathbf{R}^{d}$

$$
(|\xi|+|\eta|) / 2 \leq(|\xi-\eta|+|\eta|) \leq 2(|\xi|+|\eta|),
$$

it follows from (3) and (4) with $\delta=0,(2)$, and (11) that

$$
\begin{aligned}
|e(s)|_{L^{2}(\Omega)}^{2} & +\int_{0}^{s} \int_{\Omega}[|\nabla u|+|\nabla e|]^{p-2}|\nabla e|^{2} \\
\leq & C \int_{0}^{s} \int_{\Omega}[|\nabla u|+|\nabla e|]^{p-2}|\nabla e|\left|\nabla\left(u-v^{h}\right)\right| \\
& +C\left(\left|u_{0}^{h}\right|_{W^{1, p}(\Omega)}\right)\left[\int_{0}^{s}\left|u-v^{h}\right|_{L^{2}(\Omega)}^{2}\right]^{1 / 2}+C\left|u_{0}-u_{0}^{h}\right|_{L^{2}(\Omega)}^{2}
\end{aligned}
$$


The desired result (12) then follows from (6).

Remark 3.1. Equation (12) is the parabolic analogue of the key abstract error bound for the steady state case proved in [2]: for any $v^{h} \in V^{h}$

$$
\begin{aligned}
& \int_{\Omega}\left[|\nabla u|+\left|\nabla\left(u-u^{h}\right)\right|\right]^{p-2}\left|\nabla\left(u-u^{h}\right)\right|^{2} \\
& \leq C \int_{\Omega}\left[|\nabla u|+\left|\nabla\left(u-v^{h}\right)\right|\right]^{p-2}\left|\nabla\left(u-v^{h}\right)\right|^{2} .
\end{aligned}
$$

This result was proved in [2] using Lemma 2.2 and by exploiting the associated minimisation properties of $u$ and $u^{h}$. Tyukthin [15] and Chow [5], using the restricted version of this lemma as proved in [6] (see Remark 2.1), obtain (16) with the $|\nabla u|$ term missing from the right-hand side in the case $p \in(1,2]$ and from the left-hand side in the case $p \in(2, \infty)$. It is the presence of this term that leads to our optimal error bounds in the steady state case as discussed in the introduction.

The key difference in the parabolic case is the absence of the corresponding minimisation properties for $u$ and $u^{h}$. This is overcome by Lemma 2.3, which of course can be used in the steady state case as well, thus removing the need for there to be a corresponding minimisation principle to obtain improved error bounds.

Before proving explicit error bounds for the semidiscrete approximation, we prove an abstract error bound for a fully discrete approximation.

4. Fully discrete approximation. We now consider the following fully discrete approximation of $(P)$, the backward Euler discretisation applied to $\left(P^{h}\right)$ :

$\left(P^{h, \Delta t}\right)$ Let $\Delta t=T / N$, then for $n=1 \rightarrow N$, find $U^{n} \in V^{h}$ such that

$$
\begin{aligned}
\int_{\Omega} \frac{U^{n}-U^{n-1}}{\Delta t} v^{h}+\int_{\Omega}\left|\nabla U^{n}\right|^{p-2} \nabla U^{n} \cdot \nabla v^{h} & =\int_{\Omega} f^{n} v^{h} \quad \forall v^{h} \in V^{h}, \\
U^{0} & =u_{0}^{h},
\end{aligned}
$$

where $f^{n}(\cdot) \equiv f(\cdot, n \Delta t)$.

It is easy to establish the existence of a unique solution to $\left(P^{h, \Delta t}\right)$ (see, for example, [16]), and we have also the following analogue of Lemmas 2.1 and 3.1.

LEMMA 4.1. Under assumptions $(A)$ the unique solution $\left\{U^{n}\right\}_{n=0}^{N}$ of $\left(P^{h, \Delta t}\right)$ is such that

$$
\begin{gathered}
\Delta t \sum_{n=1}^{N}\left|\frac{U^{n}-U^{n-1}}{\Delta t}\right|_{L^{2}(\Omega)}^{2}+\max _{n=1 \rightarrow N}\left|U^{n}\right|_{W^{1, p}(\Omega)}^{p} \\
\leq C(p)\left[\Delta t \sum_{n=1}^{N}\left|f^{n}\right|_{L^{2}(\Omega)}^{2}+\left|u_{0}^{h}\right|_{W^{1, p}(\Omega)}^{p}\right] .
\end{gathered}
$$

Proof. From the convexity of $|\cdot|^{p}$, see (4), we have that for all $\xi, \eta \in \mathbf{R}^{d}$

$$
|\eta|^{p} \geq|\xi|^{p}+p|\xi|^{p-2} \xi \cdot(\eta-\xi) \text {. }
$$

The result (18) follows immediately by choosing $v^{h} \equiv\left(U^{n}-U^{n-1}\right) / \Delta t$ in the first equation of $\left(P^{h, \Delta t}\right)$, summing from $n=1 \rightarrow M, M=1 \rightarrow N$, and noting (18). 
For the purposes of the error analysis it is convenient to introduce

$$
\begin{gathered}
U(t) \equiv \frac{\left(t-t_{n-1}\right)}{\Delta t} U^{n}+\frac{\left(t_{n}-t\right)}{\Delta t} U^{n-1} \quad t \in\left[t_{n-1}, t_{n}\right] n=1 \rightarrow N, \\
\widehat{U}(t) \equiv U^{n} \quad t \in\left(t_{n-1}, t_{n}\right] n=1 \rightarrow N
\end{gathered}
$$

and

$$
\widehat{f}(t) \equiv f^{n} \quad t \in\left(t_{n-1}, t_{n}\right] \quad n=1 \rightarrow N,
$$

where $t_{n} \equiv n \Delta t$. Then $\left(P^{h, \Delta t}\right)$ can be restated: for almost every $t \in(0, T]$

$$
\begin{aligned}
\int_{\Omega} U_{t} v^{h}+\int_{\Omega}|\nabla \widehat{U}|^{p-2} \nabla \widehat{U} \cdot \nabla v^{h} & =\int_{\Omega} \widehat{f} v^{h} \quad \forall v^{h} \in V^{h}, \\
U(0) & =u_{0}^{h} .
\end{aligned}
$$

It follows from (18) that

$$
\begin{aligned}
& \left|U_{t}\right|_{L^{2}\left(0, T ; L^{2}(\Omega)\right)}^{2}+|U|_{C\left([0, T] ; W^{1, p}(\Omega)\right)}^{p}+|\widehat{U}|_{L^{\infty}\left(0, T ; W^{1, p}(\Omega)\right)}^{p} \\
& \quad \leq C(p)\left[\Delta t \sum_{n=1}^{N}\left|f^{n}\right|_{L^{2}(\Omega)}^{2}+\left|u_{0}^{h}\right|_{W^{1, p}(\Omega)}^{p}\right]
\end{aligned}
$$

and hence that

$$
\begin{gathered}
|U-\widehat{U}|_{L^{2}\left(0, T ; L^{2}(\Omega)\right)}^{2} \equiv \sum_{n=1}^{N} \int_{t_{n-1}}^{t_{n}}\left(t_{n}-t\right)^{2}\left|U_{t}\right|_{L^{2}(\Omega)}^{2} \\
\leq C\left(|f|_{C\left([0, T] ; L^{2}(\Omega)\right)},\left|u_{0}^{h}\right|_{W^{1, p}(\Omega)}\right)(\Delta t)^{2}
\end{gathered}
$$

As in [16] we make the the following extra assumption on the data:

$$
f:[0, T] \rightarrow L^{2}(\Omega) \text { is Lipschitz continuous. }
$$

ThEOREM 4.2. Under assumptions $(A)$ and $(B)$ the unique solutions $u^{h}$ and $U$ of $\left(P^{h}\right)$ and $\left(P^{h, \Delta t}\right)$, respectively, are such that

$$
\begin{aligned}
\left|u^{h}-U\right|_{C\left([0, T] ; L^{2}(\Omega)\right)}^{2}+ & \int_{0}^{T} \int_{\Omega}\left[\left|\nabla u^{h}\right|+\left|\nabla\left(u^{h}-\widehat{U}\right)\right|\right]^{p-2}\left|\nabla\left(u^{h}-\widehat{U}\right)\right|^{2} \\
& \leq C\left(\left|u_{0}^{h}\right|_{W^{1, p}(\Omega)}\right) \Delta t .
\end{aligned}
$$

Proof. Setting $E \equiv u^{h}-U$ and $\widehat{E} \equiv u^{h}-\widehat{U}$, we have from $\left(P^{h}\right)$ and (22), (23) on choosing $v^{h} \equiv \widehat{E}$ that for any $s \in(0, T]$,

$$
\begin{aligned}
& \frac{1}{2}|E(s)|_{L^{2}(\Omega)}^{2}+\int_{0}^{s} \int_{\Omega}\left(\left|\nabla u^{h}\right|^{p-2} \nabla u^{h}-|\nabla \widehat{U}|^{p-2} \nabla \widehat{U}\right) \cdot \nabla \widehat{E} \\
& \equiv \int_{0}^{s} \int_{\Omega}\left[-E_{t}(\widehat{U}-U)+(f-\widehat{f}) \widehat{E}\right] \leq C\left(\left|u_{0}^{h}\right|_{W^{1, p}(\Omega)}\right) \Delta t
\end{aligned}
$$

where we have noted (11), (24), (25), and assumption $(B)$. The desired result, (26), then follows from applying (4) and (14) to the left-hand side of the above equation. 
5. Error bounds. In this section we deduce explicit error bounds from the abstract bounds $(12)$ and (26). Let $\Omega^{h}$ be an approximation to $\Omega$ defined by $\overline{\Omega^{h}} \equiv$ $\cup_{\tau \in T^{h}} \bar{\tau}$, where $T^{h}$ is a triangulation of $\Omega^{h}$ into regular $d$-simplices, each of maximum diameter bounded above by $h$. Let $\left\{P_{j}\right\}_{j=1}^{J}$ be the vertices associated with this triangulation. We assume that (i) $P_{j} \in \partial \Omega^{h} \Rightarrow P_{j} \in \partial \Omega$ and (ii) $\Omega$ is convex so that $\Omega^{h} \subseteq \Omega$.

Associated with $T^{h}$ are the finite dimensional spaces

$$
S^{h} \equiv\left\{\chi \in C\left(\overline{\Omega^{h}}\right):\left.\chi\right|_{\tau} \text { is linear } \forall \tau \in T^{h}\right\}
$$

and

$$
S_{0}^{h} \equiv\left\{\chi \in C(\bar{\Omega}):\left.\chi\right|_{\overline{\Omega^{h}}} \in S^{h} \text { and }\left.\chi\right|_{\bar{\Omega} \backslash \overline{\Omega^{h}}} \equiv 0\right\}
$$

Let $\pi_{h}: C\left(\overline{\Omega^{h}}\right) \rightarrow S^{h}$ denote the interpolation operator such that for any $w \in C\left(\overline{\Omega^{h}}\right)$ $\pi_{h} w \in S^{h}$ satisfies $\left(\pi_{h} w\right)\left(P_{j}\right)=w\left(P_{j}\right) j=1 \rightarrow J$. We recall the following standard approximation results. For $m=0$ or 1 and for all $\tau \in T^{h}$ we have that:

(a) for $q, s \in[1, \infty]$, provided $W^{2, s}(\Omega) \subset W^{m, q}(\Omega)$

$$
\left|w-\pi_{h} w\right|_{W^{m, q}(\tau)} \leq C h^{2-m+d\left(\frac{1}{q}-\frac{1}{s}\right)}|w|_{W^{2, s}(\tau)} \quad \forall w \in W^{2, s}(\tau)
$$

and

(b) for $q>d$

$$
\left|w-\pi_{h} w\right|_{W^{m, q}(\tau)} \leq C h^{1-m}|w|_{W^{1, q}(\tau)} \quad \forall w \in W^{1, q}(\tau) .
$$

In (30) we noted the imbedding $W^{2,1}(\tau) \subset C(\bar{\tau})$ (see, for example, [8, p. 300]).

As $S_{0}^{h} \subset W^{1, \infty}(\Omega)$, we can choose $V^{h} \equiv S_{0}^{h}$. For ease of exposition we make the following assumption:

$$
\text { if } p \in(1,2) \text {, then } u_{0} \in W^{2, p}(\Omega) \text {. }
$$

It immediately follows from assumptions $(A)$ and $(C)$ and $(30),(31)$ that one can choose

$$
u_{0}^{h} \equiv \pi_{h} u_{0} \quad \text { and } \quad\left|u_{0}^{h}\right|_{W^{1, p}(\Omega)} \leq C .
$$

THEOREM 5.1. Under assumptions $(A)$ and $(C)$ the unique solutions $u$ and $u^{h}$ of $(P)$ and $\left(P^{h}\right)$, with $V^{h} \equiv S_{0}^{h}$ and $u_{0}^{h} \equiv \pi_{h} u_{0}$, are such that we have the following:

(a) if $p \in(1,2)$ and $u \in L^{2}\left(0, T ; W^{2, p}(\Omega)\right)$, then

$$
\left|u-u^{h}\right|_{C\left([0, T] ; L^{2}\left(\Omega^{h}\right)\right)}^{2}+\left\|u-u^{h}\right\|_{L^{2}\left(0, T ; W^{1, p}\left(\Omega^{h}\right)\right)}^{2} \leq C h^{p}
$$

(b) if $p \in(2, \infty)$ and $u \in L^{2}\left(0, T ; H^{2}(\Omega)\right) \cap L^{\infty}\left(0, T ; W^{1, \infty}(\Omega)\right)$, then

$$
\left|u-u^{h}\right|_{C\left([0, T] ; L^{2}\left(\Omega^{h}\right)\right)}^{2}+\left\|u-u^{h}\right\|_{L^{p}\left(0, T ; W^{1, p}\left(\Omega^{h}\right)\right)}^{p} \leq C h^{2} .
$$

Proof. Under the assumptions for both (a) and (b) we can choose $v^{h} \equiv \pi_{h} u$ in (12). Since $v^{h} \in S_{0}^{h} \Rightarrow v^{h} \equiv 0$ on $\Omega \backslash \Omega^{h}$, it also follows that (13) and hence (12) hold with $\Omega$ replaced by $\Omega^{h}$. Finally, on setting $e \equiv u-u^{h}$ and taking into consideration equations (30)-(32), we have the following inequalities: 
Case (a):

(35)

$$
\begin{aligned}
|e|_{C\left([0, T] ; L^{2}\left(\Omega^{h}\right)\right)}^{2}+|e|_{L^{2}\left(0, T ; W^{1, p}\left(\Omega^{h}\right)\right)}^{2} & \\
\leq & |e|_{C\left([0, T] ; L^{2}\left(\Omega^{h}\right)\right)}^{2}+C \int_{0}^{T}\left\{\int_{\Omega^{h}}\left([|\nabla u|+|\nabla e|]^{-1}[|\nabla u|+|\nabla e|]\right)^{(p-2) p / 2}|\nabla e|^{p}\right\}^{2 / p} \\
\leq & |e|_{C\left([0, T] ; L^{2}\left(\Omega^{h}\right)\right)}^{2}+C\left(|u|_{L^{\infty}\left(0, T ; W^{1, p}\left(\Omega^{h}\right)\right)},\left|u^{h}\right|_{L^{\infty}\left(0, T ; W^{1, p}\left(\Omega^{h}\right)\right)}\right) \\
& \times \int_{0}^{T} \int_{\Omega^{h}}[|\nabla u|+|\nabla e|]^{p-2}|\nabla e|^{2} \\
\leq & C \int_{0}^{T} \int_{\Omega^{h}}\left[|\nabla u|+\left|\nabla\left(u-\pi_{h} u\right)\right|\right]^{p-2}\left|\nabla\left(u-\pi_{h} u\right)\right|^{2} \\
& +C\left[\left|u-\pi_{h} u\right|_{L^{2}\left(0, T ; L^{2}\left(\Omega^{h}\right)\right)}+\left|u_{0}-\pi_{h} u_{0}\right|_{L^{2}\left(\Omega^{h}\right)}^{2}\right] \\
\leq & C\left[\left|u-\pi_{h} u\right|_{L^{p}\left(0, T ; W^{1, p}\left(\Omega^{h}\right)\right)}^{p}+\left|u-\pi_{h} u\right|_{L^{2}\left(0, T ; L^{2}\left(\Omega^{h}\right)\right)}+\left|u_{0}-\pi_{h} u_{0}\right|_{L^{2}\left(\Omega^{h}\right)}^{2}\right] \\
\leq & C h^{p}+C h^{2+d\left(\frac{1}{2}-\frac{1}{p}\right)}+C h^{2\left[2+d\left(\frac{1}{2}-\frac{1}{p}\right)\right]} \\
\leq & C h^{p}
\end{aligned}
$$

where we have applied a Holder inequality and noted (2), (11), and that $p \geq d / 2 \Rightarrow$ $2+d\left(\frac{1}{2}-\frac{1}{p}\right) \geq p$.

Case (b):

$$
\begin{aligned}
& |e|_{C\left([0, T] ; L^{2}\left(\Omega^{h}\right)\right)}^{2}+|e|_{L^{p}\left(0, T ; W^{1, p}\left(\Omega^{h}\right)\right)}^{p} \\
& \leq|e|_{C\left([0, T] ; L^{2}\left(\Omega^{h}\right)\right)}^{2}+\int_{0}^{T} \int_{\Omega^{h}}[|\nabla u|+|\nabla e|]^{p-2}|\nabla e|^{2} \\
& \leq C\left[\left|u-\pi_{h} u\right|_{L^{2}\left(0, T ; H^{1}\left(\Omega^{h}\right)\right)}^{2}+\left|u-\pi_{h} u\right|_{L^{2}\left(0, T ; L^{2}\left(\Omega^{h}\right)\right)}+\left|u_{0}-\pi_{h} u_{0}\right|_{L^{2}\left(\Omega^{h}\right)}^{2}\right] \\
& \leq C h^{2} .
\end{aligned}
$$

Finally, we note from a Poincaré inequality that for all $r, s \in(1, \infty)$, for all $w \in L^{r}\left(0, T ; W_{0}^{1, s}(\Omega)\right)$, and for all $w_{1}^{h}, w_{2}^{h} \in L^{r}\left(0, T ; S_{0}^{h}\right)$, that

$$
\begin{array}{rl}
\left\|w-w_{1}^{h}\right\|_{L^{r}\left(0, T ; W^{1, s}\left(\Omega^{h}\right)\right) \leq}^{r} & C\left\|w-w_{2}^{h}\right\|_{L^{r}\left(0, T ; W^{1, s}\left(\Omega^{h}\right)\right)}^{r} \\
& +C\left|w-w_{1}^{h}\right|_{L^{r}\left(0, T ; W^{1, s}\left(\Omega^{h}\right)\right)}^{r} .
\end{array}
$$

The desired result, (33), then follows from (35) and (37), with $w \equiv u, w_{1}^{h} \equiv u^{h}$ and $w_{2}^{h} \equiv \pi_{h} u$. Similarly, (34) follows from bounds (37) and (37) and noting that $\left\|u-\pi_{h} u\right\|_{L^{p}\left(0, T ; W^{1, p}\left(\Omega^{h}\right)\right)}^{p} \leq C\left\|u-\pi_{h} u\right\|_{L^{2}\left(0, T ; H^{1}\left(\Omega^{h}\right)\right)}^{2}$.

THEOREM 5.2. Under assumptions $(A),(B)$, and $(C)$, the unique solutions $u$ and $U$ of $(P)$ and $\left(P^{h, \Delta t}\right)$, with $V^{h} \equiv S_{0}^{h}$ and $u_{0}^{h} \equiv \pi_{h} u_{0}$, are such that we have the following:

(a) if $p \in(1,2)$ and $u \in L^{2}\left(0, T ; W^{2, p}(\Omega)\right)$, then

$$
|u-U|_{C\left([0, T] ; L^{2}\left(\Omega^{h}\right)\right)}^{2}+\|u-\widehat{U}\|_{L^{2}\left(0, T ; W^{1, p}\left(\Omega^{h}\right)\right)}^{2} \leq C\left(h^{p}+\Delta t\right) ;
$$

(b) if $p \in(2, \infty)$ and $u \in L^{2}\left(0, T ; H^{2}(\Omega)\right) \cap L^{\infty}\left(0, T ; W^{1, \infty}(\Omega)\right)$, then

$$
|u-U|_{C\left([0, T] ; L^{2}\left(\Omega^{h}\right)\right)}^{2}+\|u-\widehat{U}\|_{L^{p}\left(0, T ; W^{1, p}\left(\Omega^{h}\right)\right)}^{p} \leq C\left(h^{2}+\Delta t\right) .
$$


Proof. Similar to the proof of Theorem 5.1, (26) yields the following outcomes: Case (a):

$$
\begin{aligned}
\mid u^{h}- & \left.U\right|_{C\left([0, T] ; L^{2}\left(\Omega^{h}\right)\right)} ^{2}+\left\|u^{h}-\widehat{U}\right\|_{L^{2}\left(0, T ; W^{1, p}\left(\Omega^{h}\right)\right)}^{2} \\
\leq & \left|u^{h}-U\right|_{C\left([0, T] ; L^{2}\left(\Omega^{h}\right)\right)}^{2}+C\left(\left|u^{h}\right|_{L^{\infty}\left(0, T ; W^{1, p}\left(\Omega^{h}\right)\right)},|\widehat{U}|_{L^{\infty}\left(0, T ; W^{1, p}\left(\Omega^{h}\right)\right)}\right) \\
& \times \int_{0}^{T} \int_{\Omega^{h}}\left[\left|\nabla u^{h}\right|+\left|\nabla\left(u^{h}-\widehat{U}\right)\right|\right]^{p-2}\left|\nabla\left(u^{h}-\widehat{U}\right)\right|^{2} \\
\leq & C \Delta t,
\end{aligned}
$$

where we have noted (11) and (24).

Case (b):

$$
\begin{aligned}
& \left|u^{h}-U\right|_{C\left([0, T] ; L^{2}\left(\Omega^{h}\right)\right)}^{2}+\left\|u^{h}-\widehat{U}\right\|_{L^{p}\left(0, T ; W^{1, p}\left(\Omega^{h}\right)\right)}^{p} \\
& \quad \leq\left|u^{h}-U\right|_{C\left([0, T] ; L^{2}\left(\Omega^{h}\right)\right)}^{2}+\int_{0}^{T} \int_{\Omega^{h}}\left[\left|\nabla u^{h}\right|+\left|\nabla\left(u^{h}-\widehat{U}\right)\right|\right]^{p-2}\left|\nabla\left(u^{h}-\widehat{U}\right)\right|^{2} \\
& \quad \leq C \Delta t .
\end{aligned}
$$

Hence the desired results, (38) and (39), follow from combining (33) with (40), and (34) with (41), respectively.

In order to obtain $h^{2}$ in place of $h^{p}$ in the error bounds (33) and (38) for the case $p \in(1,2)$, we exploit the presence of the $|\nabla u|$ term on the right-hand side in the abstract error bound (12) (see Remark 3.1).

THEOREM 5.3. Under assumptions $(A)$ and $(C)$ the unique solutions $u$ and $u^{h}$ of $(P)$ and $\left(P^{h}\right)$, with $V^{h} \equiv S_{0}^{h}$ and $u_{0}^{h} \equiv \pi_{h} u_{0}$, are such that if $p \in(1,2)$ and $u \in L^{p}\left(0, T ; C^{2,(2-p) / p}(\bar{\Omega}) \cap W^{3,1}(\Omega)\right) \cap L^{2}\left(0, T ; H^{2}(\Omega)\right)$, then

$$
\left|u-u^{h}\right|_{C\left([0, T] ; L^{2}\left(\Omega^{h}\right)\right)}^{2}+\left\|u-u^{h}\right\|_{L^{2}\left(0, T ; W^{1, p}\left(\Omega^{h}\right)\right)}^{2} \leq C h^{2} .
$$

Furthermore, under the additional assumption $(B)$ the unique solution $U$ of $\left(P^{h, \Delta t}\right)$, with $V^{h} \equiv S_{0}^{h}$ and $u_{0}^{h} \equiv \pi_{h} u_{0}$, is such that

$$
|u-U|_{C\left([0, T] ; L^{2}\left(\Omega^{h}\right)\right)}^{2}+\|u-\widehat{U}\|_{L^{2}\left(0, T ; W^{1, p}\left(\Omega^{h}\right)\right)}^{2} \leq C\left(h^{2}+\Delta t\right) .
$$

Proof. From (35) we have that

$$
\begin{aligned}
\mid u- & \left.u^{h}\right|_{C\left([0, T] ; L^{2}\left(\Omega^{h}\right)\right)} ^{2}+\left|u-u^{h}\right|_{L^{2}\left(0, T ; W^{1, p}\left(\Omega^{h}\right)\right)}^{2} \\
\leq & C \int_{0}^{T} \int_{\Omega^{h}}\left[|\nabla u|+\left|\nabla\left(u-\pi_{h} u\right)\right|\right]^{p-2}\left|\nabla\left(u-\pi_{h} u\right)\right|^{2} \\
& +C\left[\left|u-\pi_{h} u\right|_{L^{2}\left(0, T ; L^{2}\left(\Omega^{h}\right)\right)}+\left|u_{0}-\pi_{h} u_{0}\right|_{L^{2}\left(\Omega^{h}\right)}^{2}\right] \\
\leq & C h^{2}+C \int_{0}^{T} \int_{\Omega^{h}}\left[|\nabla u|+\left|\nabla\left(u-\pi_{h} u\right)\right|\right]^{p-2}\left|\nabla\left(u-\pi_{h} u\right)\right|^{2} .
\end{aligned}
$$

We now adapt an argument used in [2] for the steady state case. For almost every $t \in(0, T) u(t) \in C^{2,(2-p) / p}(\bar{\Omega})$ it follows from (30) that for all $\tau \in T^{h}$ and for all $x \in \bar{\tau}$

$$
\begin{aligned}
\left|\nabla\left(u-\pi_{h} u\right)(x, t)\right| & \leq C h|H[u](t)|_{L^{\infty}(\tau)} \\
& \leq C h H[u](x, t)+C h^{2 / p}|u(t)|_{C^{2,(2-p) / p}(\bar{\tau})}
\end{aligned}
$$


where $H[u]$ is the Euclidean norm of the Hessian of $u$. It is easy to check that the function $\Psi(\sigma) \equiv(a+\sigma)^{p-2} \sigma^{2}$ with $a \geq 0$ is increasing on $[0, \infty)$ and hence that $\Psi\left(\left|\sigma_{1}+\sigma_{2}\right|\right) \leq 2\left[\Psi\left(\left|\sigma_{1}\right|\right)+\Psi\left(\left|\sigma_{2}\right|\right)\right]$ for all $\sigma_{1}, \sigma_{2} \in \mathbf{R}$. Therefore, it follows from (45) that

$$
\begin{aligned}
\int_{0}^{T} \int_{\Omega^{h}} & \left.|| \nabla u|+| \nabla\left(u-\pi_{h} u\right) \mid\right]^{p-2}\left|\nabla\left(u-\pi_{h} u\right)\right|^{2} \\
\leq & C h^{2} \int_{0}^{T} \int_{\Omega^{h}}[|\nabla u|+C h H[u]]^{p-2}(H[u])^{2} \\
& +C h^{4 / p} \int_{0}^{T} \int_{\Omega^{h}}\left[|\nabla u|+C h^{2 / p}|u(t)|_{C^{2,(2-p) / p}(\bar{\tau})}\right]^{p-2}|u(t)|_{C^{2,(2-p) / p}(\bar{\tau})}^{2} \\
\leq & C h^{2} \int_{0}^{T}|u(t)|_{C^{2,(2-p) / p}(\bar{\Omega})}^{p}+C h^{2} \int_{0}^{T} \int_{\Omega}|\nabla u|^{p-2}(H[u])^{2} .
\end{aligned}
$$

Setting $w_{i} \equiv u_{x_{i}}$ we have that

$$
\begin{aligned}
\int_{0}^{T} \int_{\Omega}|\nabla u|^{p-2}(H[u])^{2} & \leq C \int_{0}^{T} \int_{\Omega}\left[\sum_{i=1}^{d}\left(w_{i}^{2}\right)\right]^{(p-2) / 2} \sum_{i=1}^{d}\left|\nabla w_{i}\right|^{2} \\
& \leq C \sum_{i=1}^{d}\left[\int_{0}^{T} \int_{\Omega}\left|w_{i}\right|^{p-2}\left|\nabla w_{i}\right|^{2}\right] .
\end{aligned}
$$

In addition we have for $g \in L^{p}\left(0, T ; W^{2,1}(\Omega)\right)$ and for $i=1 \rightarrow d$ that

$$
\begin{aligned}
\int_{0}^{T} \int_{\Omega}|g|^{p-2}\left|g_{x_{i}}\right|^{2} \equiv \frac{1}{p-1} \int_{0}^{T} \int_{\Omega}\left[\operatorname{sign}(g)|g|^{p-1}\right]_{x_{i}} g_{x_{i}} \\
\equiv \frac{1}{p-1} \int_{0}^{T}\left\{\int_{\partial \Omega}\left[\operatorname{sign}(g)|g|^{p-1}\right] g_{x_{i}} n_{i}-\int_{\Omega}\left[\operatorname{sign}(g)|g|^{p-1}\right] g_{x_{i} x_{i}}\right\} \\
\quad<\infty
\end{aligned}
$$

where $n_{i}$ is the $i$ th component of the outward unit normal to $\partial \Omega$ and we have applied the trace inequality $\|\cdot\|_{L^{1}(\partial \Omega)} \leq C\|\cdot\|_{W^{1,1}(\Omega)}$. Combining (44), (46), (47), and (48) and noting (37) yields the desired result (42). Combining (42) and (40) yields the desired result (43).

Remark 5.1. Finally we note that if there exists a region

$$
\Omega_{\epsilon} \equiv\{x \in \Omega:|\nabla u(x, t)| \geq \epsilon \text { for almost every } \mathrm{t} \in(0, T)\},
$$

then one can improve on the error bound for the gradient of $u$ in the case where $p \in(2, \infty)$. With $\Omega_{\epsilon}^{h} \equiv \Omega_{\epsilon} \cap \Omega^{h}$ and under the assumptions of Theorem 5.1(b) we have on adapting $(37)$ that

$$
\epsilon^{p-2}\left|u-u^{h}\right|_{L^{2}\left(0, T ; H^{1}\left(\Omega_{\epsilon}^{h}\right)\right)}^{2} \leq \int_{0}^{T} \int_{\Omega^{h}}\left[|\nabla u|+\left|\nabla\left(u-u^{h}\right)\right|\right]^{p-2}\left|\nabla\left(u-u^{h}\right)\right|^{2} \leq C h^{2} .
$$

6. Regularity. In this final section we discuss the regularity requirements on $u$ in order for the error bounds, derived in the previous section, to hold. Unfortunately, such results are not available at present in the literature, but it is an active area of 
research worldwide. We discuss first the steady state problem with a general Dirichlet boundary condition.

Given $p \in(1, \infty), f \in L^{2}(\Omega)$, and $g \in W^{1, p}(\Omega)$, find $u \in W^{1, p}(\Omega)$ such that $u=g$ on $\partial \Omega$ and

$$
\int_{\Omega}|\nabla u|^{p-2} \nabla u \cdot \nabla v=\int_{\Omega} f v \quad \forall v \in V .
$$

It is easily deduced (see [6], for example) that there exists a unique solution to (49) and

$$
\|u\|_{W^{1, p}(\Omega)} \leq C\left[|f|_{L^{2}(\Omega)}^{1 /(p-1)}+\|g\|_{W^{1, p}(\Omega)}\right] .
$$

In one dimension and for the radially symmetric problem, it is a simple matter to establish some sharp regularity results for the solution $u$.

Lemma 6.1. If $d=1$ or $\Omega \equiv\left\{x \in \mathbf{R}^{2}:|x|<C\right\}$, with $g, C \in \mathbf{R}$, and $f(x) \equiv F(|x|)$, then the unique solution $u$ of (49) is such that the following holds.

If $p \in(1,2)$, then

$$
\begin{gathered}
d=1 \Rightarrow\|u\|_{H^{2}(\Omega)} \leq C\left[|f|_{L^{2}(\Omega)}^{1 /(p-1)}+|g|_{L^{\infty}(\Omega)}\right] \\
d=2 \Rightarrow\|u\|_{W^{2, p}(\Omega)} \leq C\left[|f|_{L^{2}(\Omega)}^{1 /(p-1)}+|g|\right] \\
d=2 \& f \in L^{q}(\Omega), q>2 ; \Rightarrow u \in H^{2}(\Omega) ; \\
f \in W^{1, q}(\Omega) ; q \geq 1 \text { if } d=1, q>1 \text { if } d=2 ; \Rightarrow u \in W^{3,1}(\Omega) .
\end{gathered}
$$

If $p \in(1,3 / 2]$, then

$$
f \in C^{0, \beta}(\bar{\Omega}), \beta \in[0,1], \Rightarrow u \in C^{2, \beta}(\bar{\Omega}) .
$$

If $p \in[3 / 2,2)$, then

$$
f \in C^{0, \beta}(\bar{\Omega}), \beta \in[0,(2-p) /(p-1)], \Rightarrow u \in C^{2, \beta}(\bar{\Omega}) .
$$

If $p \in(2, \infty)$, then

$$
\begin{aligned}
& f \in C(\bar{\Omega}), f(F) \text { changes sign only a finite number of times, } \\
& \Rightarrow u \in W^{2,1}(\Omega) \\
& f \in L^{\infty}(\Omega) \Rightarrow u \in C^{1,1 /(p-1)}(\bar{\Omega}) .
\end{aligned}
$$

Proof. The results (53)-(58) are established in [2]. The results (51) and (52) follow in a similar way. For example, if $d=1$ it is easily deduced that

$(59)\|u\|_{W^{1, \infty}(\Omega)} \leq C\left[|f|_{L^{2}(\Omega)}^{1 /(p-1)}+|g|_{L^{\infty}(\Omega)}\right] \quad$ and $\quad\left|u_{x x}\right|=\left[\left|u_{x}\right|^{2-p}|f|\right] /(p-1)$.

Therefore, combining the above yields the result (51). Similar arguments yield (52). 
By choosing $f \equiv F$ to be a constant, it can be seen that (56) and (58) are sharp. A similar limit to the regularity of $u$ for $p \in(1,3 / 2)$ can also be established. Hence the unique solution of (49) has limited regularity for infinitely smooth data $f, g$, and $\Omega$. We note that for some data, e.g., $d=2$ and $f \equiv F$ constant, one can improve on (57) by establishing that $u \in H^{2}(\Omega)$.

For the general two-dimensional steady state problem (49) Lieberman [9] has proved that if $\partial \Omega \in C^{1, \alpha}, g$ is a trace of a function $\in C^{1, \gamma}(\bar{\Omega})$ for $\alpha, \gamma \in(0,1)$, and $f \in L^{\infty}(\Omega)$, then $u \in C^{1, \beta}(\bar{\Omega})$ for some $\beta \in(0,1)$. Some local $H^{2}$ regularity results were established in [14]. Furthermore, sharp local regularity results in Holder as well as Sobolev norms for the case $f \equiv 0$ were established in [7] using the powerful theory of quasiregular mappings. Unfortunately, global regularity results on the second and third derivatives of $u$, required for our finite element error bounds, do not exist in the literature.

Recently we established the following result.

LemmA 6.2. If either (i) $\partial \Omega \in C^{2}$ and $g \in H^{2}(\Omega)$ or (ii) $\Omega$ is convex and $g=0$, then the unique solution $u$ of (49) is such that

$$
\begin{gathered}
p \in(1,2) \& f \in L^{q}(\Omega), \quad q>2, \Rightarrow u \in H^{2}(\Omega), \\
p \in(2, \infty) \& f \equiv 0 \Rightarrow u \in H^{2}(\Omega) .
\end{gathered}
$$

Proof. For a full proof, see [11]. A key step in the proof is the establishment of the following result:

$$
\|u\|_{H^{2}(\Omega)} \leq C\left[\left.\left.|| \nabla u\right|^{2-p} f\right|_{L^{2}(\Omega)}+\|g\|_{H^{2}(\Omega)}\right] .
$$

The above is the analogue of (59) and is based on an $H^{2}(\Omega)$ a priori estimate for the solution of a linear elliptic equation in the plane with only $L^{\infty}(\Omega)$ coefficients. Unfortunately, $W^{2, p}(\Omega)$ estimates of this type, in which $\|\cdot\|_{H^{2}(\Omega)}$ and $\|\cdot\|_{L^{2}(\Omega)}$ in $(62)$ are replaced by $\|\cdot\|_{W^{2, p}(\Omega)}$ and $\|\cdot\|_{L^{p}(\Omega)}$, are not available in the literature.

Using the sharp local regularity results of [7] and maximum principles we established in [12] the following result.

LemMA 6.3. Let $p \in(1,2), \partial \Omega \in C^{2,1}, f \equiv 0$, and $g \in W^{3, r}(\Omega) \cap C^{2,(2-p) / p}(\bar{\Omega})$, $r \in(1, p]$. In addition, let $g$ be such that its tangential derivative along $\partial \Omega, g_{\tau}$ satisfies $\left|g_{\tau}\left(x_{0}\right)\right|>0$, if $x_{0} \in \partial \Omega$ and $g\left(x_{0}\right)$ is not a global extremum of $g$ on $\partial \Omega$. Then the unique solution $u$ of $(49)$ is such that $u \in W^{3, r}(\Omega) \cap C^{2,(2-p) / p}(\bar{\Omega})$.

Therefore, from the above results we see that for some data $f, g$, and $\Omega$ we have that $u \in W^{3,1}(\Omega) \cap C^{2,(2-p) / p}(\bar{\Omega})$ if $p \in(1,2)$ and $u \in H^{2}(\Omega) \cap W^{1, \infty}(\Omega)$ if $p \in(2, \infty)$. Hence our optimal order error bounds for the continuous piecewise linear finite element approximation of (49) hold (see $\S 1$ ).

We now discuss the parabolic problem $(P)$. Chen and DiBenedetto in [4] have proved that if $\partial \Omega \in C^{1, \alpha}, \alpha \in(0,1)$, and $f \in L^{\infty}\left(0, T ; L^{\infty}(\Omega)\right)$, then $\nabla u \in C^{\beta}(\bar{\Omega} \times$ $(0, T])$ for some $\beta \in(0,1)$. However, as for the steady state case, the regularity results that we require are not available in the literature. We have the following results.

LEMMA 6.4. Let $p \in(1,2)$ and the assumptions $(A)$ and $(B)$ hold. In addition we assume that $\nabla \cdot\left(\left|\nabla u_{0}\right|^{p-2} \nabla u_{0}\right) \in L^{2}(\Omega)$. Then the unique solution $u$ of $(P)$ is such that $u_{t} \in C\left([0, T], L^{2}(\Omega)\right) \cap L^{2}\left(0, T ; W^{1, p}(\Omega)\right)$.

Proof. We note that under the above assumptions Wei [16] proved in the case where $p \in(2, \infty)$ that $u \in C\left([0, T], W^{1, p}(\Omega)\right)$ and $u_{t} \in C\left([0, T], L^{2}(\Omega)\right)$. It is a simple 
matter to extend the proof to $p \in(1, \infty)$. Then formally differentiating $(P)$ with respect to $t$ yields

$$
u_{t t}=\nabla \cdot\left[|\nabla u|^{p-2} \nabla u_{t}+(p-2)|\nabla u|^{p-4}\left(\nabla u . \nabla u_{t}\right) \nabla u\right]+f_{t} .
$$

Multiplying the above by $u_{t}$, integrating over $\Omega \times(0, s), s \in(0, T]$, and performing integration by parts yields

$$
\begin{aligned}
\frac{1}{2} \int_{0}^{s} \frac{d}{d t} \int_{\Omega}\left(u_{t}\right)^{2} & +\int_{0}^{s} \int_{\Omega}\left[|\nabla u|^{p-2}\left|\nabla u_{t}\right|^{2}+(p-2)|\nabla u|^{p-4}\left(\nabla u . \nabla u_{t}\right)^{2}\right] \\
& =\int_{0}^{s} \int_{\Omega} f_{t} u_{t} .
\end{aligned}
$$

Hence it follows that for all $s \in(0, T]$

$$
\left|u_{t}(s)\right|_{L^{2}(\Omega)}^{2}+(p-1) \int_{0}^{s} \int_{\Omega}|\nabla u|^{p-2}\left|\nabla u_{t}\right|^{2} \leq C\left[\left|u_{t}(0)\right|_{L^{2}(\Omega)}^{2}+\left|f_{t}\right|_{L^{1}\left(0, s ; L^{2}(\Omega)\right)}\right] .
$$

It follows from a Holder inequality, as $p \in(1,2)$, that

$$
\left|u_{t}\right|_{L^{2}\left(0, T ; W^{1, p}(\Omega)\right)}^{2} \leq C|u|_{L^{\infty}\left(0, T ; W^{1, p}(\Omega)\right)}^{2-p} \int_{0}^{T} \int_{\Omega}|\nabla u|^{p-2}\left|\nabla u_{t}\right|^{2} .
$$

Justifying the formal argument above in the standard way it follows from (63), (64), our assumptions, and (2) that $u_{t} \in L^{2}\left(0, T ; W^{1, p}(\Omega)\right)$.

LEMMA 6.5. Let the assumptions of Lemma 6.4 hold. If $d=1$, then the unique solution $u$ of $(P)$ is such that $u \in L^{2}\left(0, T ; H^{2}(\Omega)\right)$. If $\Omega \equiv\left\{x \in \mathbf{R}^{2}:|x|<C\right\}$, with $C \in \mathbf{R}, f(x) \equiv F(|x|)$ and $u_{0}(x) \equiv U_{0}(|x|)$, then the unique solution $u$ of $(P)$ is such that $u \in L^{2}\left(0, T ; W^{2, p}(\Omega)\right)$.

Proof. It follows from (51) that for almost every $t \in(0, T]$

$$
\|u(t)\|_{H^{2}(\Omega)} \leq C\left[|f(t)|_{L^{2}(\Omega)}^{1 /(p-1)}+\left|u_{t}(t)\right|_{L^{2}(\Omega)}^{1 /(p-1)}\right] .
$$

The desired result for $d=1$ then follows from our assumptions and Lemma 6.4. A similar argument using the bound (52) yields the desired result in the radially symmetric case.

LEMMA 6.6. In addition to the assumptions of Lemma 6.4, we assume that either $\Omega \in C^{2}$ or $\Omega$ is convex. Then if $p \in\left[\frac{3}{2}, 2\right)$ and $f \in L^{2}\left(0, T ; L^{2 p /(3 p-4)}(\Omega)\right)$, the unique solution $u$ of $(P)$ is such that $u \in L^{2}\left(0, T ; H^{2}(\Omega)\right)$.

Proof. For almost every $t \in(0, T]$ we have from (62) that

$$
\begin{aligned}
\|u(t)\|_{H^{2}(\Omega)} & \leq\left.\left. C|| \nabla u(t)\right|^{2-p}\left(f(t)+u_{t}(t)\right)\right|_{L^{2}(\Omega)} \\
& \leq C|u(t)|_{W^{1, p}(\Omega)}^{2-p}\left[|f(t)|_{L^{2 p /(3 p-4)}(\Omega)}+\left|u_{t}(t)\right|_{L^{2 p /(3 p-4)}(\Omega)}\right]
\end{aligned}
$$

where we have applied a Holder inequality, noting that $p>4 / 3$. The desired result then follows from our assumptions, (2), Lemma 6.4, and the observation that $W^{1, p}(\Omega) \subseteq L^{2 p /(3 p-4)}(\Omega)$ for $p \in\left[\frac{3}{2}, 2\right)$.

Above we have exhibited a general class of data which leads to the solution $u$ of $(P)$ satisfying the regularity requirements of Theorems 5.1(a) and 5.2(a). Unfortunately, we do not have similar results for the requirements of Theorems 5.1(b), 5.2(b), and 5.3. Below we just state some solutions that do achieve the required regularity. 
Consider the one-dimensional problem

$$
\begin{aligned}
u_{t} & =\left(\left|u_{x}\right|^{p-2} u_{x}\right)_{x} \quad x \in(0,1), t \in(0,1] ; \\
u(x, 0) & =u_{0}(x), \quad x \in(0,1) \\
u(0, t)=g_{1}(t), \quad u(1, t) & =g_{2}(t), \quad t \in(0,1] .
\end{aligned}
$$

If $p \in(1,2)$, then for an appropriate choice of $u_{0}, g_{1}$, and $g_{2}$

$$
u(x, t)=\left(\frac{2-p}{p-1}(3-x-t)\right)^{\frac{1-p}{p-2}}
$$

is the unique solution of (67).

If $p \in(2, \infty)$, then for an appropriate choice of $u_{0}, g_{1}$, and $g_{2}$

$$
u(x, t)= \begin{cases}0 & \text { if } x+t \in[0,1] \\ \left(\frac{p-2}{p-1}(x+t-1)\right)^{\frac{p-1}{p-2}} & \text { if } x+t \in[1,2]\end{cases}
$$

is the unique solution of $(67)$.

Setting $\Omega \equiv(0,1)$ and $T=1$, we see immediately that $(68)$ is infinitely smooth on $\bar{\Omega} \times[0, T]$ and hence satisfies the regularity requirement for Theorem 5.3. In addition, we note that $\left|u_{x}(x, t)\right| \geq C>0$ for all $(x, t) \in \bar{\Omega} \times[0, T]$ and thus (67) does not degenerate in this case.

A simple calculation yields that (69) is such that $u \in L^{\infty}\left(0, T ; W^{1, \infty}(\Omega)\right) \cap$ $L^{2}\left(0, T ; H^{2}(\Omega)\right)$, the regularity requirement for Theorems $5.1(\mathrm{~b})$ and $5.2(\mathrm{~b})$, if $p \in$ $(2,4)$. Note that $(69)$ satisfies the regularity requirement for Wei's result $(1), u \in$ $C\left([0, T] ; W^{2, p}(\Omega)\right)$ only if $p \in(2,2+\sqrt{ } 2)$. In addition, we note that (67) does degenerate in this case.

Finally, we note that alternative explicit solutions can be found in [3], where similarity solutions $u(x, t)=U(|x|, t)=\mathcal{U}(\zeta)$ with $\zeta=|x| t^{-1 / p}$ are constructed for the parabolic $p$-Laplacian, $p \in(1, \infty)$, with $f \equiv 0$. It is easily established that these solutions have essentially the same regularity for $p \in(1,2)$ and $p \in(2, \infty)$ as $(68)$ and (69), respectively.

\section{REFERENCES}

[1] C. AtKInson AND C. W. Jones, Similarity solutions in some non-linear diffusion problems and in boundary-layer flow of a pseudo plastic fluid, Quart. J. Mech. Appl. Math., 27 (1974), pp. 193-211.

[2] J. W. BARRETT AND W. B. LiU, Finite element approximation of the p-Laplacian, Math. Comp., 61 (1993), pp. 523-537.

[3] J. E. Boulllet And C. Atkinson, A generalised diffusion equation: Radial symmetries and comparison theorems, J. Math. Anal. Appl., 95 (1983), pp. 37-68.

[4] Y. Z. Chen AND E. DiBenedetto, Boundary estimates for solutions of nonlinear degenerate parabolic systems, J. Reine Angew. Math., 395 (1989), pp. 102-131.

[5] S. S. CHOW, Finite element error estimates for non-linear elliptic equations of monotone type, Numer. Math., 54 (1989), pp. 373-393.

[6] R. Glowinski AND A. MARRocCO, Sur l'approximation par éléments finis d'ordre un, et la résolution, par pénalisation-dualitié, d'une classe de problèmes de Dirichlet non linéaires, RAIRO Analyse Numérique, 2 (1975), pp. 41-76.

[7] T. IwANIEC AND J. J. MAnfRedi, Regularity of p-harmonic functions on the plane, Rev. Ma. Iberoamericana, 5 (1989), pp. 1-19.

[8] A. Kufner, O. John, AND S. FuČIK, Function Spaces, Nordhoff, Leyden, the Netherlands, 1977. 
[9] G. M. Lieberman, Boundary regularity for solutions of degenerate elliptic equations, J. Nonlinear Anal., 12 (1988), pp. 1203-1219.

[10] J. L. LiOns AND E. MAGenes, Non-Homogeneous Boundary Value Problems and Applications - Vol. I, Springer-Verlag, Berlin, 1972.

[11] W. B. LiU AND J. W. BARRETt, A remark on the regularity of the solutions of the p-Laplacian and its applications to their finite element approximation, J. Math. Anal. Appl., 178 (1993), pp. $470-487$.

[12] - A further remark on the regularity of the solutions of the p-Laplacian and its applications to their finite element approximation, J. Nonlinear Anal., 21 (1993), pp. 379-387.

[13] J. R. Philip, N-diffusion, Austral. J. Phys., 14 (1961), pp. 1-13.

[14] P. Tolksdorf, Regularity for a more general class of quasilinear elliptic equations, J. Differential Equations, 51 (1984), pp. 126-150.

[15] V. B. TYukHtin, The rate of convergence of approximation methods of solution of one-sided variational problems, Izv. Leningrad Univ. Mat. Mekh. Astronom., 13 (1982), pp. 111-113.

[16] D. WEI, Existence, uniqueness, and numerical analysis of solutions of a quasilinear parabolic problem, SIAM J. Numer. Anal., 29 (1992), pp. 484-497. 